\title{
The Study of Microchannel Plate Gain Using SIMION
}

\author{
Yifan Zhao \\ Shanghai Jianping High School, Shanghai, China \\ Email:meganzhao2002@outlook.com
}

How to cite this paper: Zhao, Y.F. (2020) The Study of Microchannel Plate Gain Using SIMION. Open Journal of Modelling and Simulation, 8, 1-17.

https://doi.org/10.4236/ojmsi.2020.81001

Received: November 19, 2019

Accepted: December 13, 2019

Published: December 16, 2019

Copyright $\odot 2020$ by author(s) and Scientific Research Publishing Inc. This work is licensed under the Creative Commons Attribution International License (CC BY 4.0).

http://creativecommons.org/licenses/by/4.0/

\section{(c) (i) Open Access}

\begin{abstract}
Microchannel plates (MCP) are widely used for particle detection. The gain of chevron MCPs is related to geometrical parameters, but no study has been done through SIMION simulation. The purpose of this study is to model a chevron MCP and its secondary emission process using SIMION and determine the relationship between microchannel plate gain, voltage, channel bias angle, and diameter. Two geometry files simulated MCP electric field and shape, and a Lua program simulated secondary emission. Simulation results showed that MCP gain is proportional to voltage, angles between 5 and 15 degrees maximize gain, and gain is inversely proportional to the diameter. This study accurately simulates a chevron MCP and yields the relationship between gain, voltage, channel bias angle, and diameter. Further studies are needed to simulate electron trajectories for improved precision.
\end{abstract}

\section{Keywords}

Microchannel Plate, SIMION, Secondary Emission, Ion Simulation

\section{Introduction}

Microchannel plates (MCP) are planar components that are widely used for particle detection [1]. MCPs share many similarities with electron multiplier in that they both amplify signals from a single particle through secondary emission. Typically, microchannel plates are made from lead glasses with a regular array of approximately 10,000,000 microchannels leading from one face to another. At the rear and front surfaces of the MCP is a metallic coating. The surfaces serve as input and output electrodes. Microchannel plates have a length to diameter ratio ranging from 40 to 100 [2]. Figure 1 shows the basic structure of a MCP. Note that microchannel plates come in different configurations: straight channel MCPs, chevron MCPs, and Z stack MCPs. This thesis intends to evaluate the ef- 
fect of applied voltage, channel bias angle, and diameter has on microchannel plate gain using SIMION. Currently, there is a consensus that the microchannel plate gain is proportional to the applied voltage, and a channel bias angle between $5^{\circ}$ and $15^{\circ}$ yields maximum gain [3]. MCP gain is proportional to length to diameter ratio [2]. Therefore, MCP gain is inversely proportional to diameter. These results generally came from experiments, and no comprehensive study of MCP gain has been done with SIMION.

\section{Model and Theory}

\subsection{Microchannel Plate Detector Overview}

A single channel microchannel plate acts as a particle amplifier. A particle entering the MCP is guaranteed to hit the wall since the channels are at an angle from the plate. This particle then causes a cascade of electrons which eventually amplify the input signal. The process of electron ejection due to input particle bombarding the MCP wall is called secondary emission. This process is modeled through a Lua program (see 3.1.2). Chevron microchannel plate consists of two single-channel MCPs rotated $180^{\circ}$ from each other. Figure 2 is a diagram of a chevron MCP. The channel bias angle is the angle between the channel axis and the vertical axis to the plate surface [4]. Figure 3 shows the channel bias angle $\theta$ in chevron MCPs. Channel bias angle allows sizeable directional change which

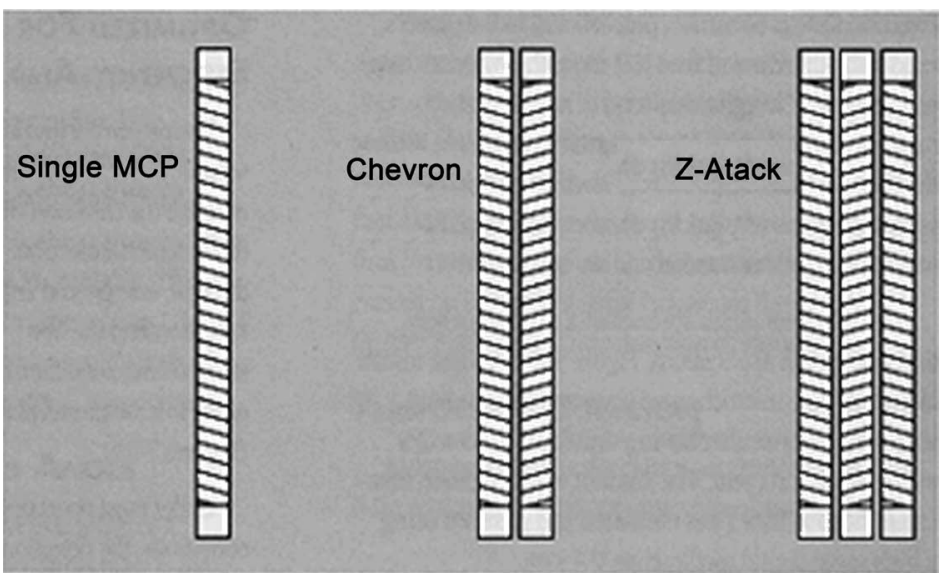

Figure 1. Typical MCP configurations [5].

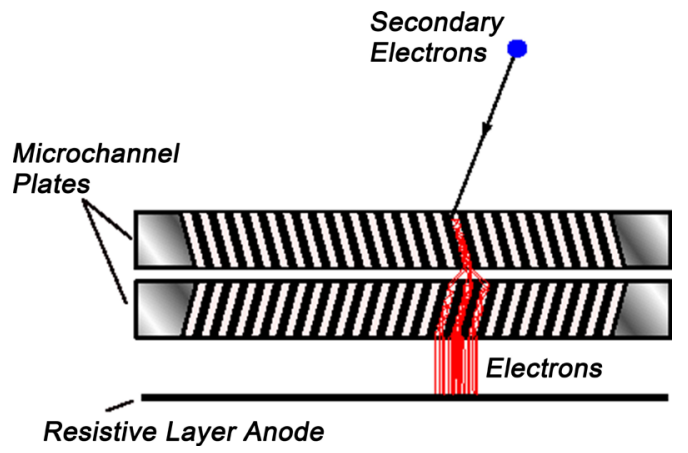

Figure 2. Chevron microchannel plate [6]. 


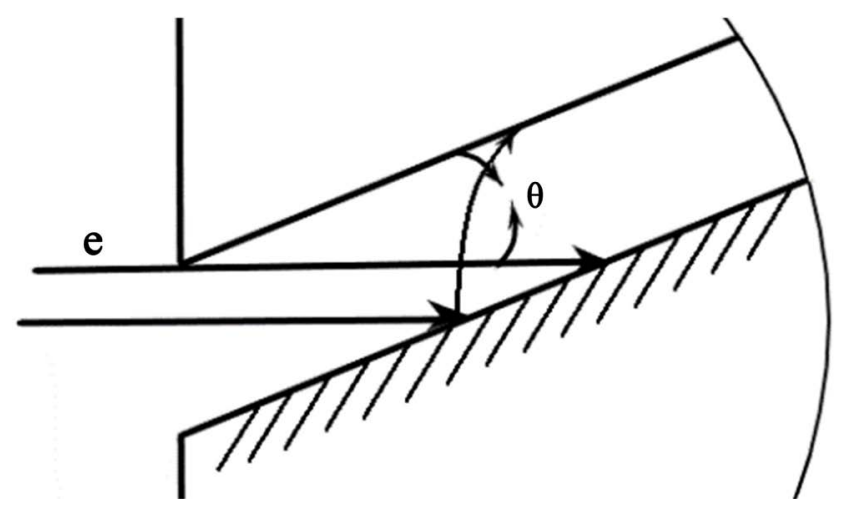

Figure 3. Channel bias angle.

inhibits ions at MCP output from reaching the input. In a chevron MCP, the two plates are usually separated by $50-150 \mu \mathrm{m}$, with a voltage applied between two plates. The features of chevron MCPs are modeled with geometry files (see 3.1.1).

In 1979, Wiza [3] modeled MCP gain with total channel voltage, the initial energy of the secondary electron, and length to diameter ratio. Wiza [3] finds that total channel voltage is proportional to gain in both single-channel and chevron MCPs. In 2017, Chen et al. [7] studied the effect of geometry parameters have on microchannel plate gain. Chen [7]'s study concluded that a channel bias angle of 12 degrees maximized MCP gain. These studies are compared with this thesis' simulation results (see section 4).

\subsection{SIMION Overview}

SIMION is an ion optics simulation program capable of modeling ion optic problems with 2D symmetry and/or 3D asymmetry and/or electrostatic and/or magnetic potential arrays. Geometry files (GEM files) are advanced SIMION features that allow users to define complex electrode or pole geometry, especially complex 3D array geometry. SIMION provides a secondary emission example that models secondary electron emission, allowing users to compute surface normal with an analytical expression [8]. This example program is modified using the Lua language.

\section{Examination of Factors Affecting Microchannel Plate Gain}

\subsection{Simulation Setup}

\subsubsection{Microchannel Plate in SIMION}

A geometry file creates the electric field applied across the chevron microchannel plate. This file is a $3000 * 150 * 50\left(x^{*} y^{*} z\right)$ planar, non-mirrored space in which resides two 3-D boxes of electrodes located at $x=290, y=0, z=0$ and $x=$ 2310, $y=0, z=0$. The boxes of electrodes have $x$-direction length two grid units, $y$-direction length 150 grid units, and $z$-direction length 50 grid units. The left box of electrodes is set at -100 volts and the right box of electrodes is adjustable. The electric field geometry file is projected onto the workbench with a millime- 
ter to grid unit ratio of 0.001 . This workbench is then saved. Another geometry file mimics one tube of a chevron microchannel plate's two plates. The geometry file defines again a planar, non-mirrored $3000 * 150 * 50\left(x^{\star} y^{\star} z\right)$ space in which there are two tubes with outer radius 12 grid units and adjustable inner radius. The left tube is at $x=300$ to $x=1298$, and the right tube is at $x=1302$ to $x=2300$. The angle between the tubes and the $x$-axis is the channel bias angle. Figure 4 is an example of the two tubes in $x$ - $y$ plane.

\subsubsection{Secondary Electron Emission in SIMION}

The simulation of secondary electron emission in SIMION utilizes the SIMION secondary emission example (see 2.2 ) with a few modifications. Firstly, SIMION secondary emission example allows users to incorporate an analytical emission for surface normal. The analytical expressions used are

$$
\begin{gathered}
n x=\left(y-10-x \cdot \tan \left(\theta^{\circ}\right)\right) \cdot \cos \left(\theta^{\circ}\right) \cdot \sin \left(\theta^{\circ}\right) \\
n y=\left(y-10-x \cdot \tan \left(\theta^{\circ}\right)\right) \cdot \cos \left(\theta^{\circ}\right) \cdot \cos \left(\theta^{\circ}\right) \\
n z=25-z
\end{gathered}
$$

for the left tube, and

$$
\begin{gathered}
n x=-\left(y-10-(2000-x) \cdot \tan \left(\theta^{\circ}\right)\right) \cdot \cos \left(\theta^{\circ}\right) \cdot \sin \left(\theta^{\circ}\right) \\
n y=-\left(y-10-(2000-x) \cdot \tan \left(\theta^{\circ}\right)\right) \cdot \cos \left(\theta^{\circ}\right) \cdot \cos \left(\theta^{\circ}\right) \\
n z=25-z
\end{gathered}
$$

for the right tube, where $n x, n y, n z$ are surface normal vectors and $\theta$ is the channel bias angle. All surface normal vectors point toward the center of the tubes. Figure 5 shows the geometry of $n x$ and $n y$, where $x$-component and $y$-component represents $n x$, ny. Figure 6 shows the geometry of $n z$ viewed from $z$-y plane.

The "process_collision_early" function is also modified. Because $(y-10-x \cdot \tan \theta)^{2}+(z-25)^{2}=$ radius of tube ${ }^{2}$, the condition for the input electron hitting the tube surface is $(y-10-(300-x) \cdot \tan \theta)^{2}+(z-25)^{2}>r^{2}$, where $r$ is inner radius. 300 is where the tube is located on the $x$-axis. If this condition holds, then the program would proceed to execute secondary emission.

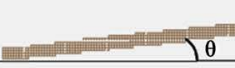

Figure 4. Chevron MCP tubes in $x$ - $y$ plane.

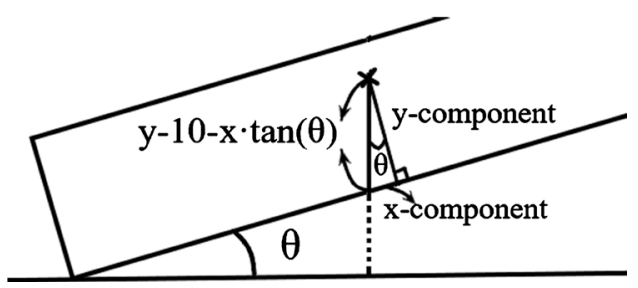

Figure 5. $n x$ and nygeometry. 
Finally, since the SIMION secondary emission example only simulates one input electron producing one secondary electron, new variables are needed to represent the total number of secondary electrons produced i.e. microchannel plate gain. Local variable "secondary_add" represents the number of electrons produced every time an electron hits the tube surface. "Secondary_add" equals probability of secondary emission times variable "secondary_amplitude". The probability of secondary emission and input energy form a Gaussian distribution, and this value is calculated and fixed. "Secondary_amplitude" scales the final gain to a reasonable number of digits. This variable is adjustable. The total gain equals number of electrons in the workbench times "secondary_add".

\subsection{Simulation with Changing Voltage in Chevron Microchannel Plate}

This simulation is to measure how microchannel plate gain changes with increasing voltage applied between the two ends of the MCP. In each trial, energy and channel bias angle are fixed. Variable "secondary_amplitude" is set so that the gain is approximately $10^{7}$, which is consistent with previous experiments by Wiza [3] and Eberhardt [9]. It is expected that MCP gain increases as voltage increases.

Trial I

Table 1 is the parameters of this trial. Voltage between $600 \mathrm{~V}$ and $2900 \mathrm{~V}$ is tested.

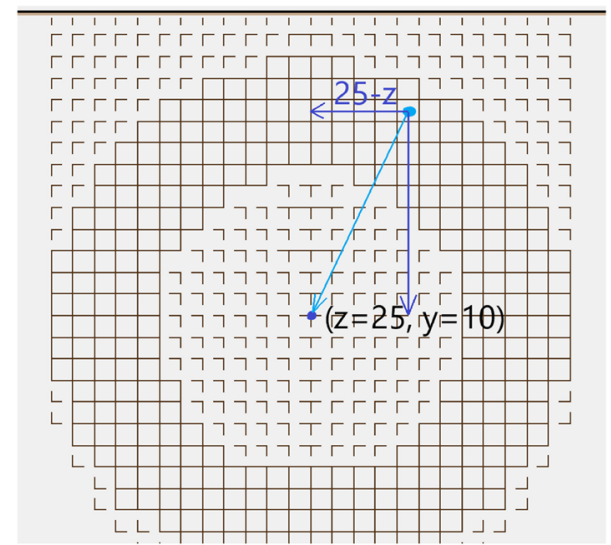

Figure 6. $n z$ geometry in $z-y$ plane.

Table 1. Parameters of voltage simulation trial I.

\begin{tabular}{cc}
\hline name & value \\
\hline Channel bias angle $(\theta)$ & $6^{\circ}$ \\
Inner radius & $7 \mathrm{gu}$ \\
Outer radius & $12 \mathrm{gu}$ \\
Energy $(E)$ & $20 \mathrm{eV}$ \\
Secondary_amplitude & 13.7
\end{tabular}




\section{Trial II}

Parameters of this trial are in Table 2.

Trial III

Table 3 is the parameters of this trial. Figure 11 shows the result of this trial. At $E=50 \mathrm{eV}$, the graph is similar to those of the previous trials. However, as Figure 12 shows, the voltage vs. gain graph at low voltage is not smooth like Figure 10 and Figure 8.

\subsection{Simulation with Changing Channel Bias Angle}

This simulation measures how microchannel plate gain changes with varying channel bias angle $\theta$. Channel bias angle is modified at the MCP geometry file and in the secondary emission program. At each energy level, three voltages are chosen: $800 \mathrm{~V}, 1500 \mathrm{~V}$, and $2200 \mathrm{~V}$ for the sake of universality. This simulation aims to find a channel bias angle that maximizes microchannel plate gain at different energy and voltage. However, angles beyond 16 degrees are not measured because the field potential instances needed to contain two tubes with bias angle larger than 16 degrees are too large for the ion optics workbench. For all three trials, microchannel plates have inner radius 7 grid units and outer radius 12 grid units. Trial I is performed at energy equals $20 \mathrm{eV}$. Trial II is performed at energy equals $40 \mathrm{eV}$ with voltage of $800 \mathrm{~V}, 1500 \mathrm{~V}$, and $2200 \mathrm{~V}$. In Trial III, energy equals $50 \mathrm{eV}$.

\subsection{Simulation with Changing Diameter in Chevron Microchannel Plate}

This simulation measures the effect microchannel plate diameter has on MCP gain. Diameter is modified by changing the radius in the condition for the input

Table 2. Parameters of voltage simulation trial II.

\begin{tabular}{cc}
\hline name & value \\
\hline Channel bias angle $(\theta)$ & $6^{\circ}$ \\
Inner radius & $7 \mathrm{gu}$ \\
Outer radius & $12 \mathrm{gu}$ \\
Energy $(E)$ & $40 \mathrm{eV}$ \\
Secondary_amplitude & 2.05 \\
\hline
\end{tabular}

Table 3. Parameters of voltage simulation trial III.

\begin{tabular}{cc}
\hline name & value \\
\hline Channel bias angle $(\theta)$ & $6^{\circ}$ \\
Inner radius & $7 \mathrm{gu}$ \\
Outer radius & $12 \mathrm{gu}$ \\
Energy $(E)$ & $50 \mathrm{eV}$ \\
Secondary_amplitude & 0.77
\end{tabular}


electron to hit the tube surface:

$(y-10-(300-x) \cdot \tan \theta)^{2}+(z-25)^{2}>$ inner radius of tube ${ }^{2}$. Diameters equal inner radius times two. Diameters between 12.8 grid units and 16.62 grid units are measured. Table 4 shows all diameters measured. According to previous studies, MCP gain is proportional to L/D (length to diameter ratio) [3]. Thus, it is expected that microchannel plate gain would fall as diameter or radius increases. Three trials are performed. Trail I is performed at energy equals $20 \mathrm{eV}$, voltage equals $2200 \mathrm{~V}$, and channel bias angle equals 6 degrees. In Trial II, energy equals $40 \mathrm{eV}$, voltage equals $2200 \mathrm{~V}$, and channel bias angle equals 6 degrees. Trial III is performed at energy equals $50 \mathrm{eV}$, voltage equals $2200 \mathrm{~V}$, and channel bias angle equals 6 degrees.

\section{Result and Discussion}

\subsection{Changing Voltage}

\section{Trial I}

Figure 7 is the result of this trial. From the graph, microchannel plate gain is proportional to voltage applied. The shape of this graph resembles the graph of an exponential function since gain increases more rapidly at higher voltage. Figure 8 shows voltage vs. gain at low voltage (below $1300 \mathrm{~V}$ ). The graph is a smooth curve with the same features as the voltage vs. gain graph for all voltages tested.

Trial II

Figure 9 is the result of this trial. At a higher energy, gain is still proportional to voltage, and the curve still resembles that of an exponential function. The same is true with voltage vs. gain at voltage below $1300 \mathrm{~V}$ (Figure 10).

Table 4. List of diameters measured.

\begin{tabular}{cc}
\hline radius $^{2}(\mathrm{gu})$ & Diameter $(\mathrm{gu})$ \\
\hline 41 & 12.8 \\
43 & 13.12 \\
45 & 13.42 \\
47 & 13.72 \\
49 & 14 \\
51 & 14.28 \\
53 & 14.56 \\
55 & 14.84 \\
57 & 15.1 \\
59 & 15.36 \\
61 & 15.62 \\
63 & 15.88 \\
65 & 16.12 \\
67 & 16.38 \\
69 & 16.62
\end{tabular}




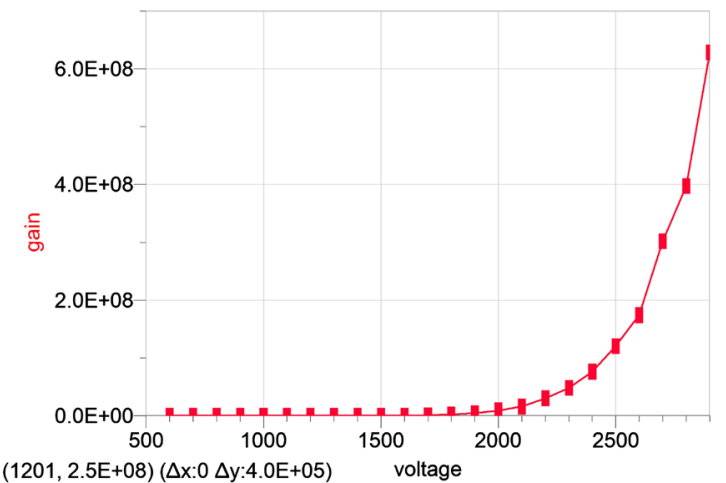

Figure 7. Voltage vs. gain $\left(E=20 \mathrm{eV}, \theta=6^{\circ}\right)$.

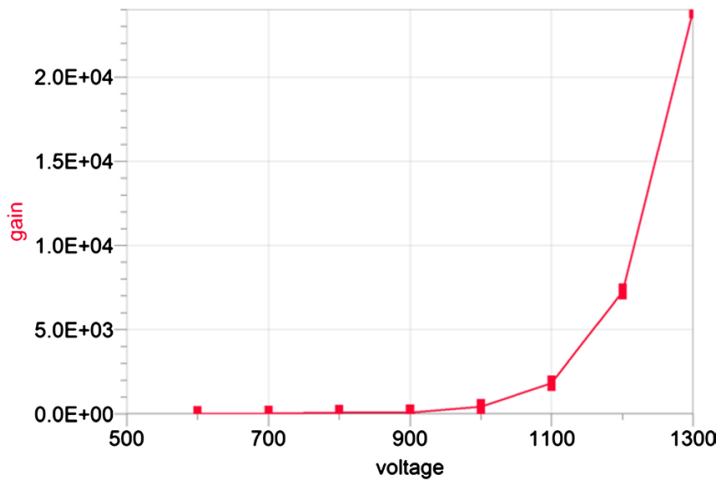

Figure 8. Voltage vs. gain at voltage below $1300 \mathrm{~V}\left(E=20 \mathrm{eV}, \theta=6^{\circ}\right)$.

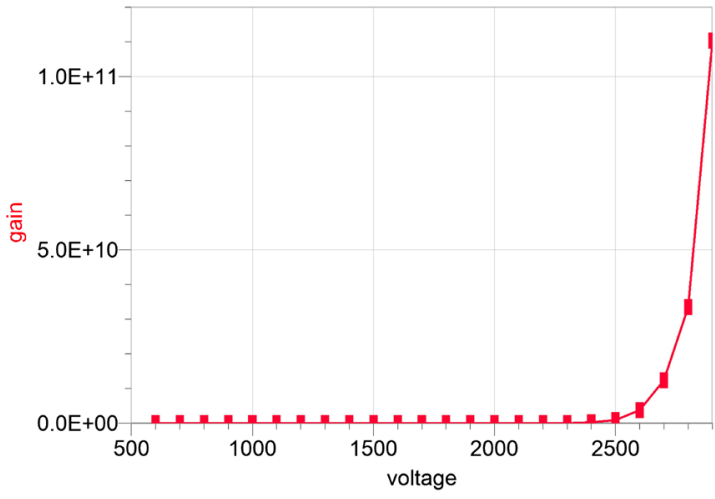

Figure 9. Voltage vs. gain $\left(E=40 \mathrm{eV}, \theta=6^{\circ}\right)$.

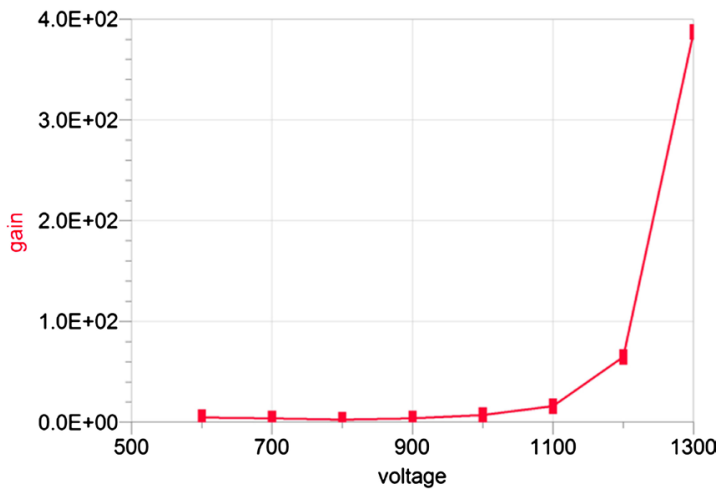

Figure 10. Voltage vs. gain at voltage below $1300 \mathrm{~V}\left(E=40 \mathrm{eV}, \theta=6^{\circ}\right)$. 


\section{Trial III}

Figure 11 shows the result of this trial. At $E=50 \mathrm{eV}$, the graph is similar to those of the previous trials. However, as Figure 12 shows, the voltage vs. gain graph at low voltage is not smooth like Figure 10 and Figure 8.

From the three trials of simulations, we observe that microchannel plate gain is proportional to the voltage applied. The voltage vs. gain curve resembles that of an exponential function i.e., the slope increases as voltage increases. The proportional relationship is consistent with studies done by Wiza [3]. The reason for such a relationship is that as the voltage applied increases, each collision happens at higher energy, thus increasing microchannel plate gain [3]. However, according to Hamamatsu [10]'s study, voltage vs. gain curves for chevron microchannel plates are bowed out (see Figure 13). The voltage vs. gain curve obtained in this thesis does not match Hamamatsu [10]'s experiment results. This problem may stem from this thesis's program's reliance on "secondary_add," the parameter in the SIMION program that approximates the additional number of electrons after each collision. Since "secondary_add" is an approximation, gain, which equals the number of electrons times "secondary_add," can be inaccurate at high voltage. This problem may be resolved by simulating the trajectories of secondary electrons produced since this simulation provides precise data on the number of electrons produced as gain.

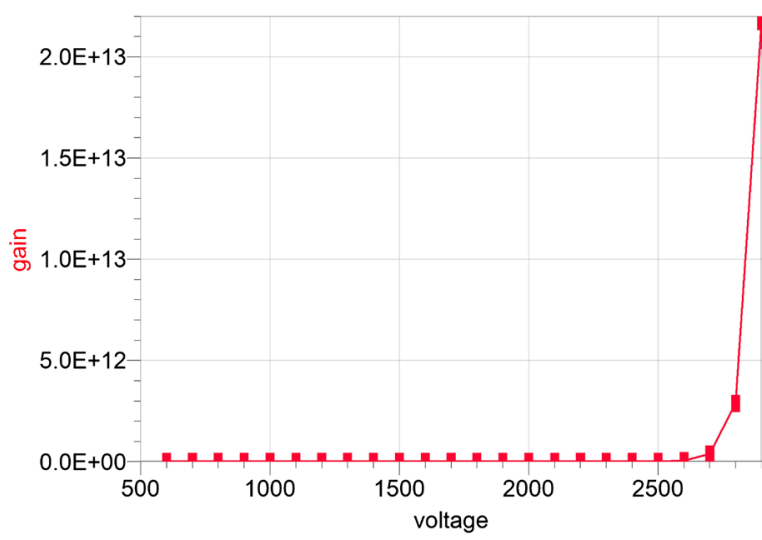

Figure 11. Voltage vs. gain $\left(E=50 \mathrm{eV}, \theta=6^{\circ}\right)$.

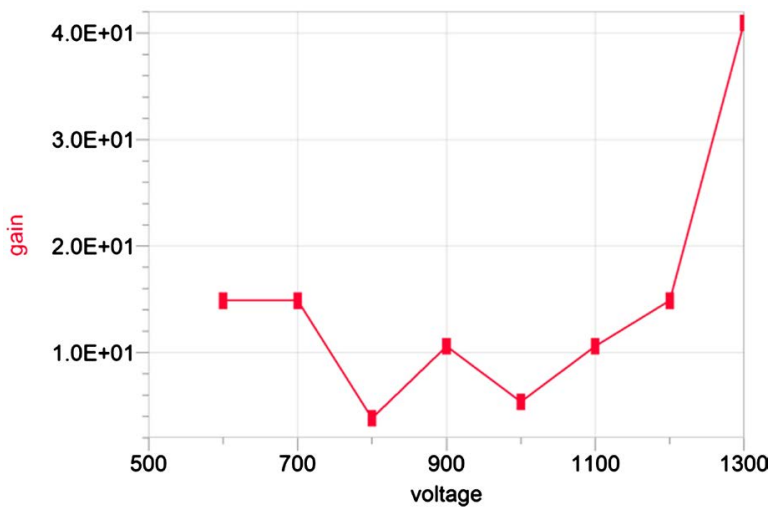

Figure 12. Voltage vs. gain at voltage below $1300 \mathrm{~V}\left(E=50 \mathrm{eV}, \theta=6^{\circ}\right)$. 


\subsection{Changing Channel Bias Angle}

\section{Trial I}

Figures 14-16 show channel bias angle vs. gain at voltage equals $800 \mathrm{~V}, 1500$ $\mathrm{V}$, and $2200 \mathrm{~V}$, respectively. From the graphs, channel bias angles that maximize gain at $V=800 \mathrm{~V}$ and $V=1500 \mathrm{~V}$ are indeterminant since gain is at maximum at an angle of 16 degrees. It cannot be determined whether larger bias angle would produce an even larger gain. At $V=2200 \mathrm{~V}$, gain decreases slightly at a channel bias angle of 16 degrees. Therefore, an angle of 15 degrees maximizes microchannel plate gain.

Trial II

Figures 17-19 show the results. In Figure 17, microchannel plate gain clearly peaks at an angle of 14 degrees. Thus, channel bias angle of 14 degrees maximize MCP gain at a voltage of $800 \mathrm{~V}$. At $V=1500 \mathrm{~V}$, channel bias angle that maximizes

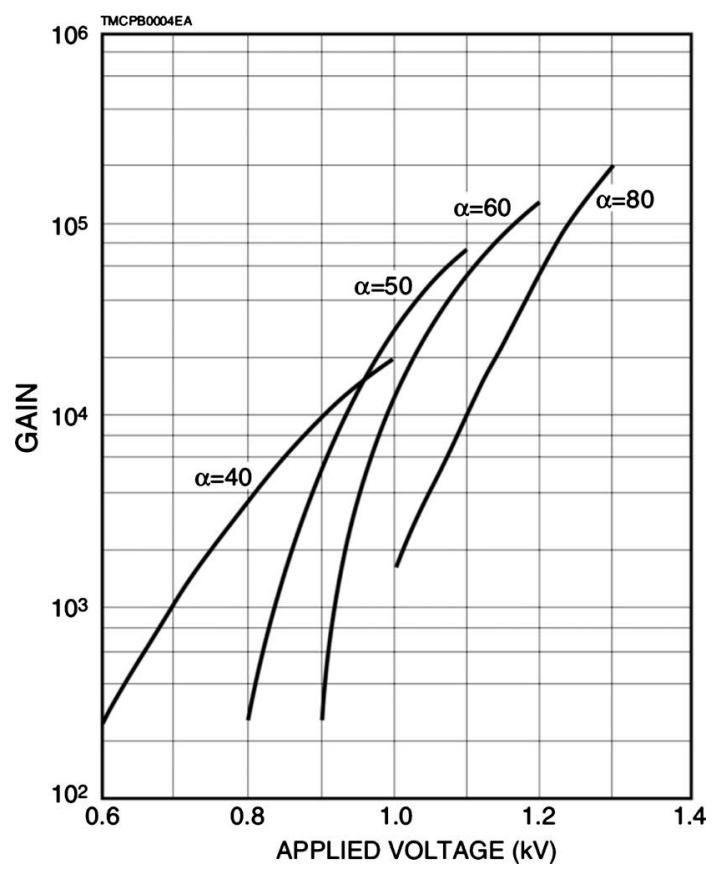

Figure 13. Voltage vs. gain [10].

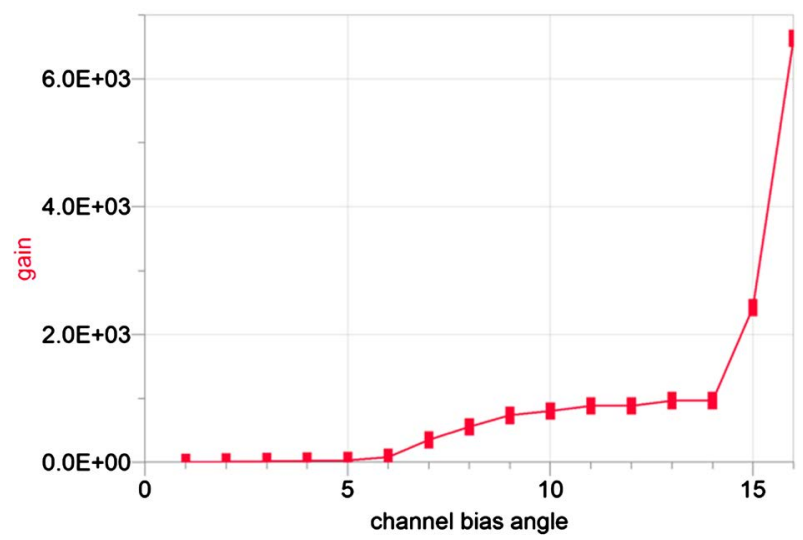

Figure 14. Channel bias angle vs. gain $(E=20 \mathrm{eV}, V=800 \mathrm{~V})$. 


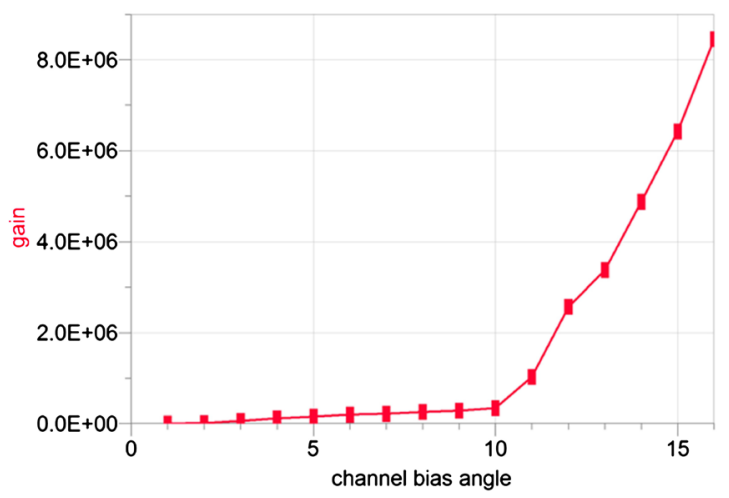

Figure 15. Channel bias angle vs. gain $(E=20 \mathrm{eV}, V=1500 \mathrm{~V})$.

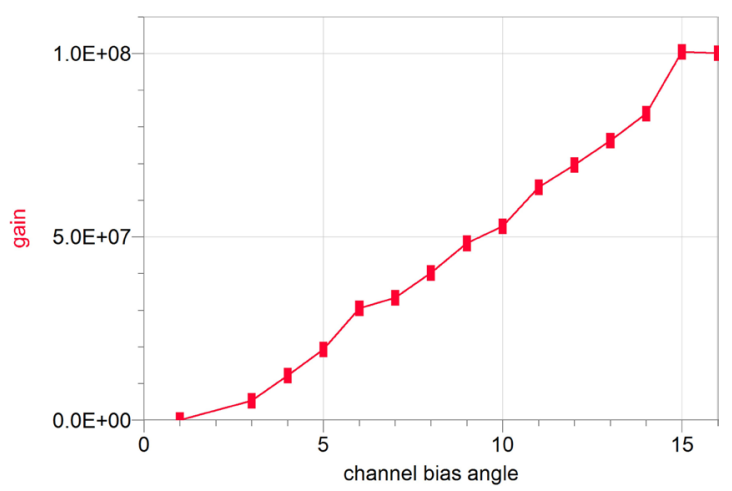

Figure 16. Channel bias angle vs. gain $(E=20 \mathrm{eV}, V=2200 \mathrm{~V})$.

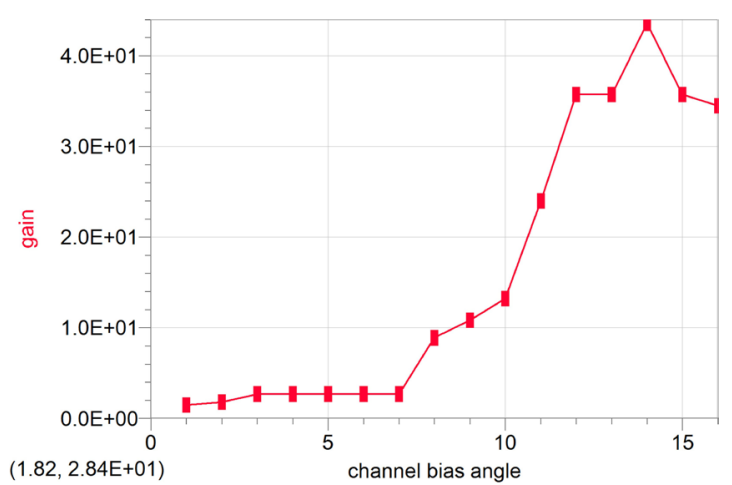

Figure 17. Channel bias angle vs. gain $(E=40 \mathrm{eV}, V=800 \mathrm{~V})$.

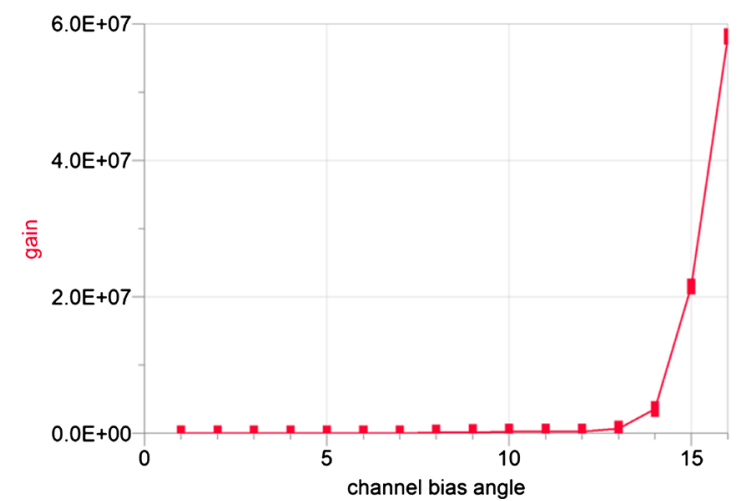

Figure 18. Channel bias angle vs. gain $(E=40 \mathrm{eV}, V=1500 \mathrm{~V})$. 
MCP gain is again indeterminant. However, since microchannel plate gain levels off at 15 and 16 degrees as shown in Figure 19, a bias angle of 15 degrees maximize MCP gain.

Trial III

Figures $20-22$ are the results at $V=800 \mathrm{~V}, 1500 \mathrm{~V}$, and $2200 \mathrm{~V}$. At $V=800 \mathrm{~V}$ and $2200 \mathrm{~V}$, angles that maximize microchannel plate gain are indeterminant as Figure 20 and Figure 22 show. At $V=1500 \mathrm{~V}$ as shown in Figure 21, MCP gain

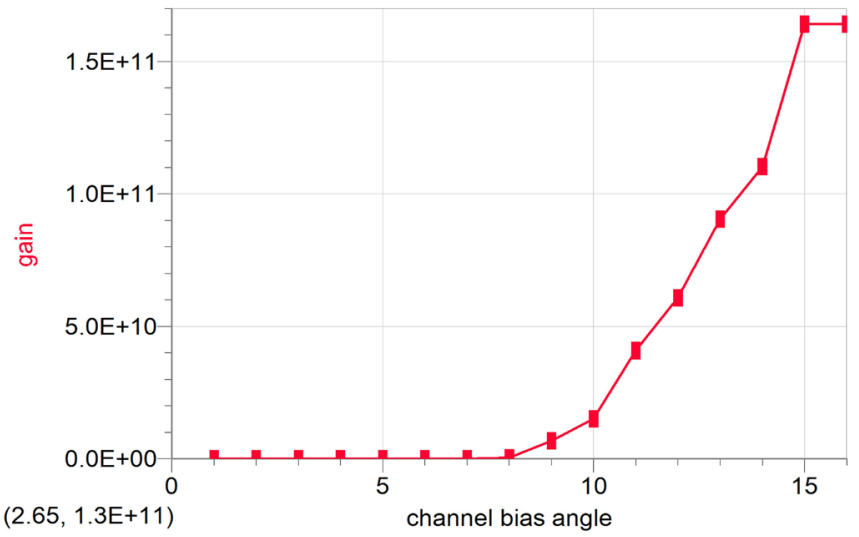

Figure 19. Channel bias angle vs. gain $(E=40 \mathrm{eV}, V=2200 \mathrm{~V})$.

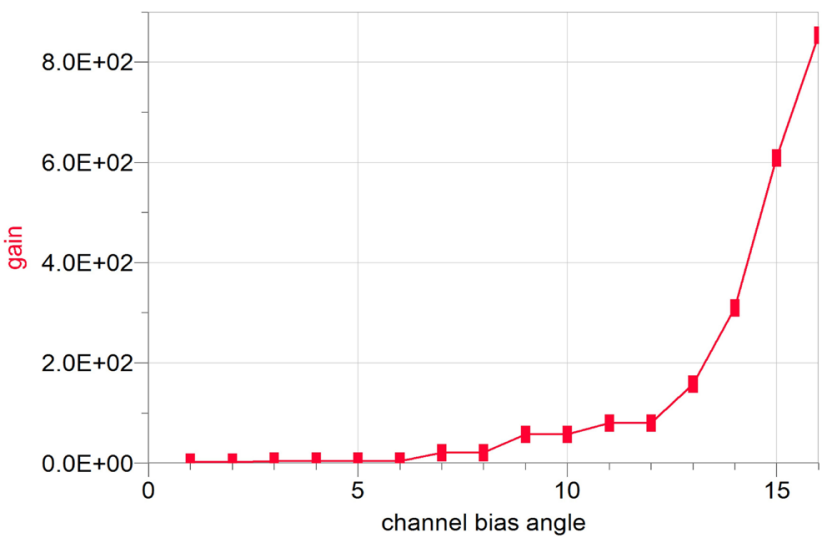

Figure 20. Channel bias angle vs. gain $(E=50 \mathrm{eV}, V=800 \mathrm{~V})$.

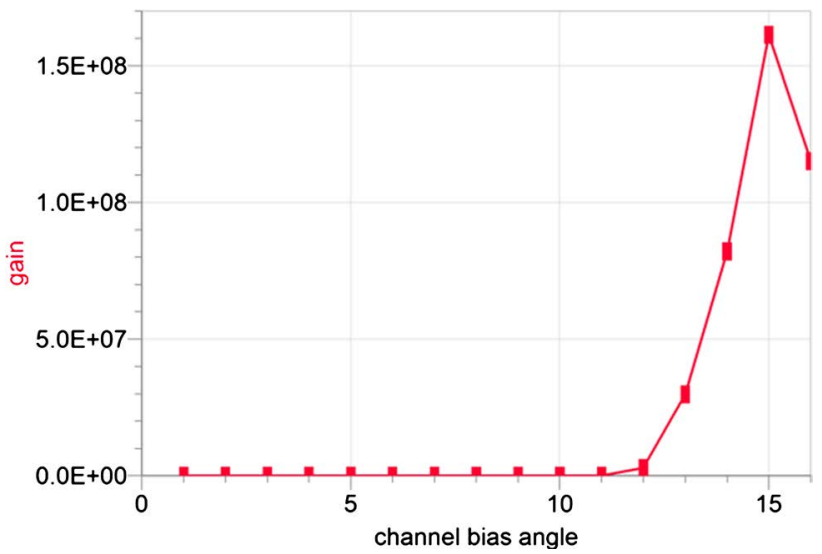

Figure 21. Channel bias angle vs. gain $(E=50 \mathrm{eV}, V=1500 \mathrm{~V})$. 
peaks at an angle of 15 degrees. Therefore, it is the channel bias angle that maximizes MCP gain.

Simulation results show that at different energies and voltages, peak gains occur at angles of 14 or 15 degrees. Table 5 lists the four bias angle that maximizes microchannel plate gain. According to Chen et al. [7]'s study, the channel bias angle between 5 and 15 maximize MCP gain. A larger angle causes a decrease in gain because the input electrons have shorter accelerating distances. The results obtained in this thesis are in accordance with well-established studies. This proves that SIMION can accurately simulate the effect of varying channel bias angle through GEM files. One limitation of this thesis is that some gain maximizing angles are indeterminant because the creation of GEM files with angles larger than 16 exceeds the maximum volume of the SIMION workbench. A possible solution is to create two GEM files, representing the left and right parts of a chevron MCP. Then, we need to create a program that retains results from the left MCP, and the results can be inputted into the right MCP.

\subsection{Changing Diameter}

Trial I

Figure 23 shows the result. As shown in the graph, MCP gain is inversely proportional to diameter except at diameter equals 12.8 and 15.88 .

Trial II

As shown in Figure 24, microchannel plate gain decreases from 1.18E+07 to below 1.0E+07. However, at diameter equals 13.42 and 15.88, MCP gain is inconsistent with the inverse relationship between diameter and gain.

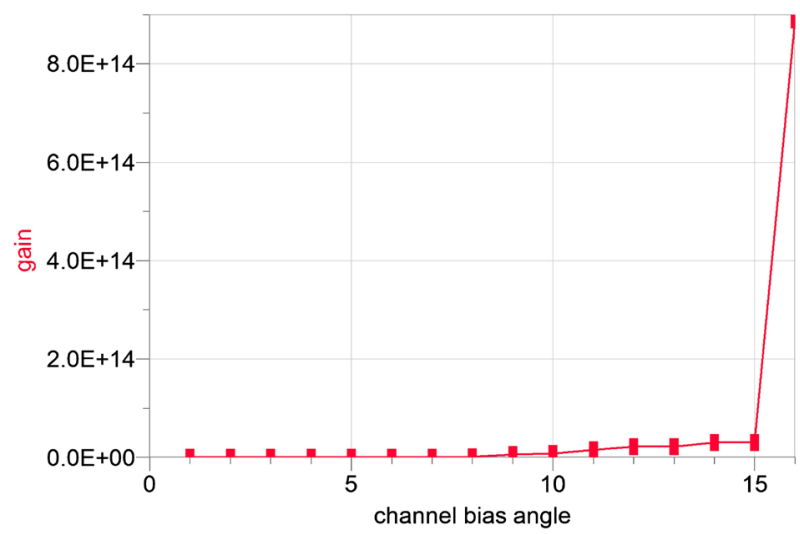

Figure 22. Channel bias angle vs. gain $(E=50 \mathrm{eV}, V=2200 \mathrm{~V})$.

Table 5. List of channel bias angle that maximizes MCP gain.

\begin{tabular}{cccc}
\hline channel bias angle $\theta\left(^{\circ}\right)$ & Energy $(\mathrm{eV})$ & Voltage $(\mathrm{V})$ & Diameter $(\mathrm{gu})$ \\
\hline 15 & 20 & 2200 & 7 \\
14 & 40 & 800 & 7 \\
15 & 40 & 2200 & 7 \\
15 & 50 & 1500 & 7 \\
\hline
\end{tabular}




\section{Trial III}

Figure 25 clearly shows the inverse relationship between microchannel plate diameter and gain.

According to simulation results at different energies, as diameter increases, microchannel plate gain decreases. According to Chen et al. [7]'s study, microchannel plate gain is proportional to the length to diameter ratio (L/D). In this thesis, the length is unchanged. Therefore, it is expected that gain is inversely proportional to diameter, which is in accordance with simulation results. This shows that SIMION can simulate the effect of changing diameter using different

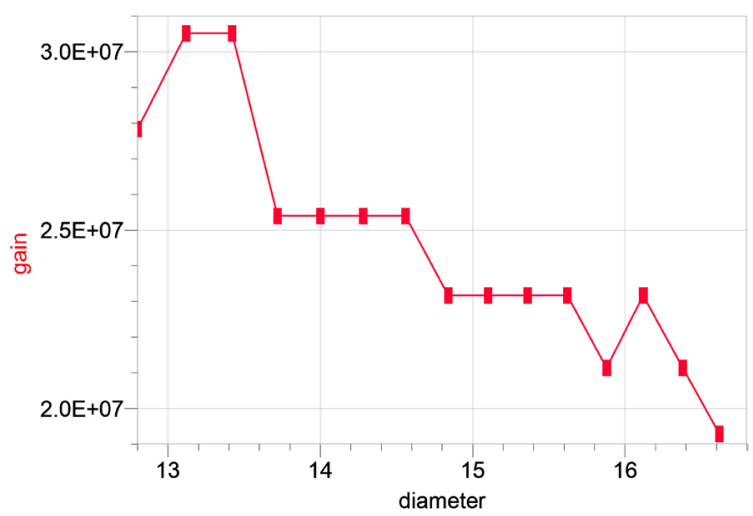

Figure 23. Diameter vs. gain $\left(E=20 \mathrm{eV}, V=2200 \mathrm{~V}, \theta=6^{\circ}\right)$.

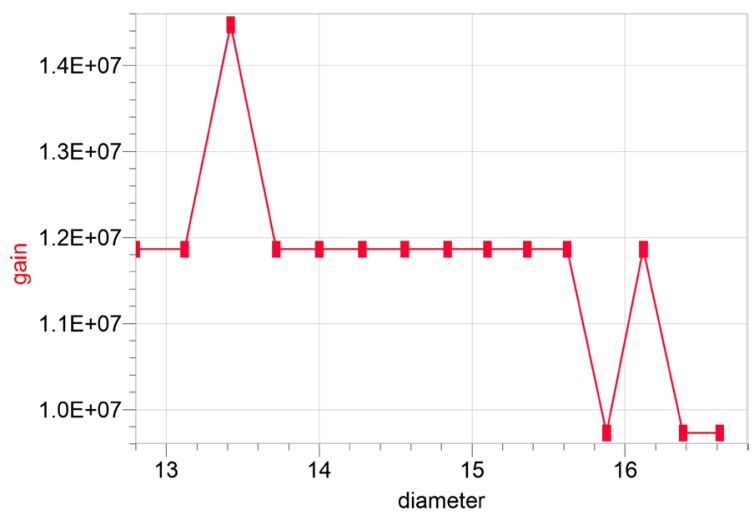

Figure 24. Diameter vs. gain $\left(E=40 \mathrm{eV}, V=2200 \mathrm{~V}, \theta=6^{\circ}\right)$.

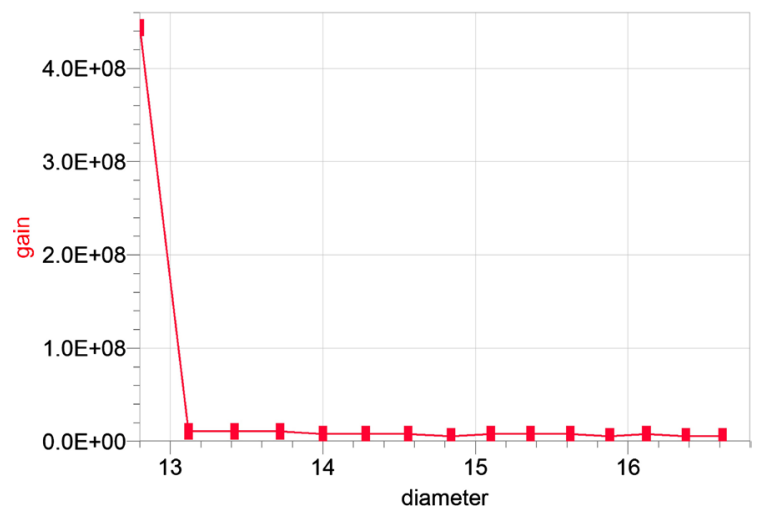

Figure 25. Diameter vs. gain $\left(E=50 \mathrm{eV}, V=2200 \mathrm{~V}, \theta=6^{\circ}\right)$. 
GEM files with accuracy. One limitation of this thesis is that although diameter vs. gain curves follow an inverse relationship in general, there exist inconsistent data points. One possible explanation is that the program used to simulate each collision that produces secondary electrons fails to take into account the change in accelerating distances in the direction perpendicular to the tube axis. This problem can be resolved by simulating the trajectories of secondary electrons, which take into account the accelerating distances.

\section{Conclusion}

Through simulation of the secondary electron emission process using SIMION, this thesis examines the relationship between microchannel plate gain and voltage, channel bias angle, and diameter. Simulation results show that microchannel plate gain is proportional to voltage, which is in accordance with well-established studies. Simulation with varying channel bias angle yields four angles between 5 and 15 degrees that maximize microchannel plate gain between 5 and 15 degrees, matching Chen et al. [7]'s study. Finally, simulations with changing diameter show that gain is inversely proportional to the diameter. This result is in accordance with Chen et al. [7]'s results: gain is proportional to the length to diameter ratio (L/D). Therefore, the simulation of a chevron microchannel plate gain using SIMION is successful. Future studies can focus on resolving the limitations of this thesis by simulating trajectories of secondary electrons produced and testing channel bias angle greater than 15 .

\section{Conflicts of Interest}

The author declares no conflicts of interest regarding the publication of this paper.

\section{References}

[1] Taylor, R.C., et al. (1983) Maximizing the Quantum Efficiency of Microchannel Plate Detectors: The Collection of Photoelectrons from the Interchannel Web Using an Electric Field. Review of Scientific Instruments, 54, 171-176. https://doi.org/10.1063/1.1137365

[2] Delmar Photonics (2008) Microchannel Plates. http://www.dmphotonics.com/MCP_MCPImageIntensifiers/microchannel_plates.h tm

[3] Wiza, J.L. (1979) Microchannel Plate Detectors. Nuclear Instruments and Methods, 162, 587-601. https://doi.org/10.1016/0029-554X(79)90734-1

[4] Fraser, G.W. (2002) The Ion Detection Efficiency of Microchannel Plates (MCPs). International Journal of Mass Spectrometry, 215, 13-30.

https://doi.org/10.1016/S1387-3806(01)00553-X

[5] Optimacorp.co (2014) Long Life Microchannel Plates. http://www.optimacorp.co.jp/BurleEO/MCP.htm

[6] Lynch, W.G., et al. (2013) Tracking Rare-Isotope Beams with Microchannel Plates. ArXiv.org. https://arxiv.org/abs/1309.2745

[7] Chen, L., et al. (2017) The Gain and Time Characteristics of Microchannel Plates in 
Various Channel Geometries. IEEE Transactions on Nuclear Science, 64, 1080-1086. https://doi.org/10.1109/TNS.2017.2676010

[8] Manura, D. and David, A.D. (2007) SIMION 8.0 User Manual. Scientific Instrument Services.

[9] Eberhardt, E.H. (1979) Gain Model for Microchannel Plates. Applied Optics, 18, 1418. https://doi.org/10.1364/AO.18.001418

[10] Hamamatsu Photonics (2019) MCP Assembly. https://www.hamamatsu.com/resources/pdf/etd/MCP_TMCP0002E.pdf 


\section{Appendix}

Field geometry file code

pa_define $(3000,150,50$, planar, non-mirrored)

locate $(290,0,0)\{$

$\mathrm{e}(1)\{$

fill $\{$ within $\{\operatorname{box} 3 d(0,0,0,2,150,50)\}\}$

\}

\}

locate $(2310,0,0)\{$

$\mathrm{e}(2)\{$

fill $\{$ within $\{\operatorname{box} 3 \mathrm{~d}(0,0,0,2,150,50)\}\}$

\}

\}

Secondary emission program code

simion.workbench_program()

simion.import("tubelib_b.lua")

-- Compute a normal vector analytically

-- at given position $(\mathrm{x}, \mathrm{y}, \mathrm{z})$ in $\mathrm{mm}$ workbench coordinates.

-- This is used by secondarylib.lua to define the surface

-- normal vector analytically.

-- This is used only if secondary_normal_mode $==2$.

function secondary_user_normal $(\mathrm{x}, \mathrm{y}, \mathrm{z})$

$\mathrm{x}=\mathrm{x} /$ ion_mm_per_grid_unit

$\mathrm{y}=\mathrm{y} /$ ion_mm_per_grid_unit

$\mathrm{z}=\mathrm{z} / \mathrm{ion} \_\mathrm{mm} \_$per_grid_unit

local nx, ny, nz

if $\mathrm{x}<=1299$ and $\mathrm{x}>299$ then -- left tube

$\mathrm{nx}=(\mathrm{y}-10-\mathrm{x} * \text { math } \tan (\operatorname{math} \cdot \operatorname{rad}(6)))^{*}$ math. $\cos (\operatorname{math} \cdot \operatorname{rad}(6)) *$ math.sin $(\operatorname{math} \cdot \operatorname{rad}(6)) ;$

ny $=-\left(y-10-x^{*} \text { math.tan }(\operatorname{math} \cdot \operatorname{rad}(6))\right)^{*}$ math $\cdot \cos (\operatorname{math} \cdot \operatorname{rad}(6))^{*}$ math $\cdot \cos (\operatorname{math} \cdot \operatorname{rad}(6))$;

$\mathrm{nz}=25-\mathrm{z}$;

elseif $x>=1300$ and $x<2300$ then--right tube

$\mathrm{nx}=-\left(\mathrm{y}-10-(2300-\mathrm{x})^{*} \text { math } \cdot \tan (\operatorname{math} \cdot \operatorname{rad}(6))\right)^{\star}$ math $\cdot \cos (\operatorname{math} \cdot \operatorname{rad}(6))^{\star}$ math $\cdot \sin (\operatorname{math} \cdot \operatorname{rad}(6))$;

$\left.\mathrm{ny}=-\left(\mathrm{y}-10-(2300-\mathrm{x})^{\star} \operatorname{math} \cdot \tan (\operatorname{math} \cdot \operatorname{rad}(6))\right)\right)^{*} \operatorname{math} \cdot \cos (\operatorname{math} \cdot \operatorname{rad}(6))^{*} \operatorname{math} \cdot \cos (\operatorname{math} \cdot \operatorname{rad}(6)) ; \mathrm{nz}=25-\mathrm{z}$;

else

-- unknown, so leave undefined, causing a splat.

end

return $\mathrm{nx}, \mathrm{ny}, \mathrm{nz}$

end 